\title{
Preparation, Characterization, and Theoretical Treatment Complexes Of Some Transition Metals with N5 Acyclic Derived From 2, 6-diacetyl pyridine
}

\author{
*Omar Hamad Shehab AL- Obaidi, Mahmmod Al-Jubori \\ ${ }^{*}$ Chemistry department, Women Education College, Al-Anbar University \\ e-mail: dromaralobaidi@yahoo.com \\ Chemistry department, College of science, Al-Mustansrya University
}

\begin{abstract}
:
A series of transition metal complexes with [2,6-diacetimino-pheylenediamine -[2,2'diyl] pyridine ]of the general formula $[\mathrm{MLCl}] \mathrm{Cl}, \mathrm{M}=\mathrm{Co}(\mathrm{II}), \mathrm{Ni}(\mathrm{II})$ and $\mathrm{Cu}(\mathrm{II})$, respectively have been synthesized and fully characterized on the bases of C.H.N.M elemental analysis, ${ }^{1} \mathrm{HNMR}$, UV-Visible and FTIR spectra, in addition the structure of complexes was characterized by magnetic moments and molar conductance in DMSO solution ,and molar ratio of metal were also determined. From the above of these studies and measurements suggest an octahedral geometry around $\mathrm{Co}$ (II), $\mathrm{Ni}$ (II) and $\mathrm{Cu}(\mathrm{II})$.
\end{abstract}

A theoretical treatment of the formation of complexes in the gas phase was studied, this was done using the HYPERCHEM-6 program for the Molecular mechanics and Semi-empirical calculations.

Keywords: Preparation, characterization, Theoretical Treatment, $\mathrm{N}_{5}$ acyclic, transition metal.

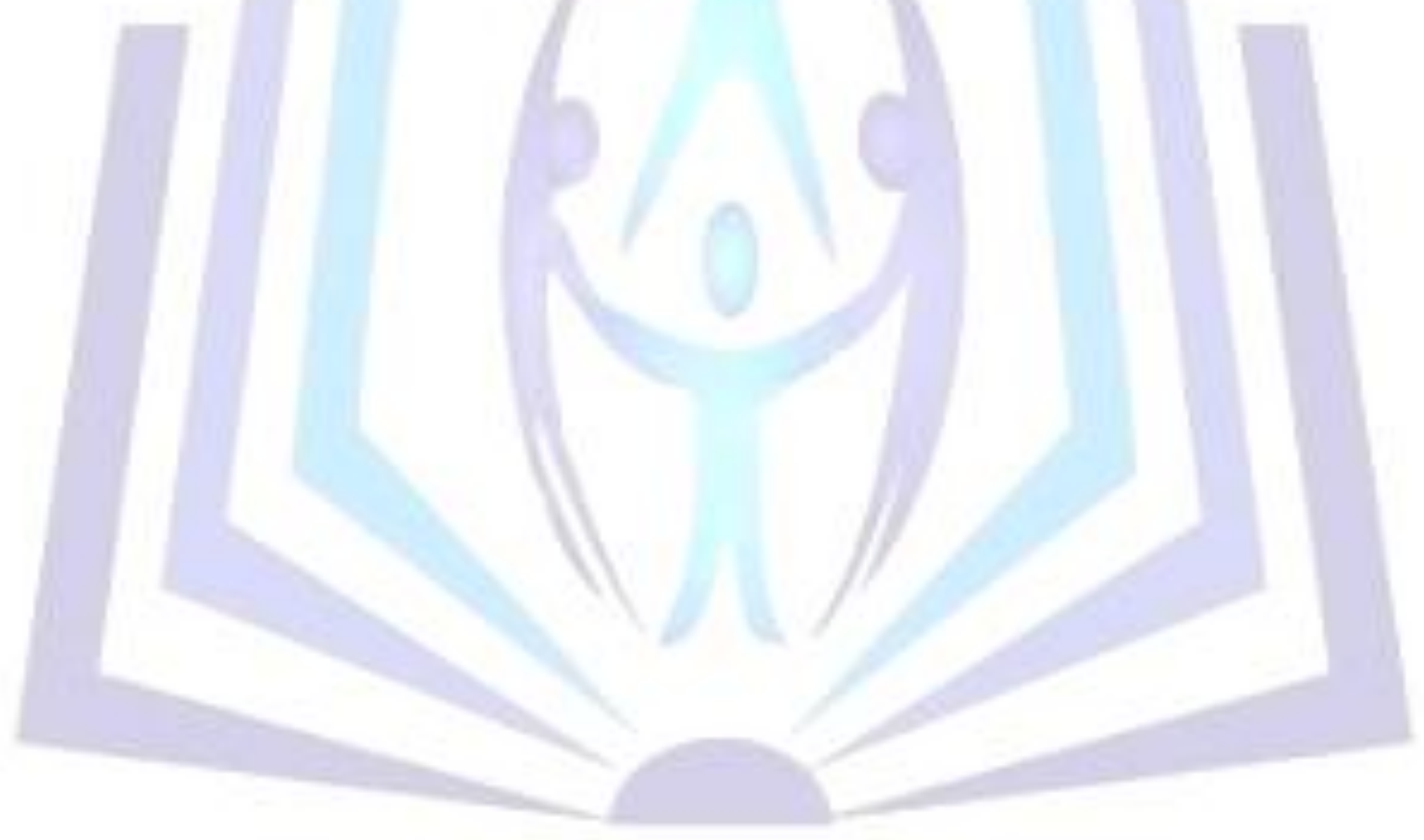

\section{Council for Innovative Research}

Peer Review Research Publishing System

Journal: Journal of Advances in Chemistry

Vol 2, No. 2

editor@cirworld.com

www.cirworld.com, member.cirworld.com 


\section{Introduction:}

Complexes of metal ions with synthetic macro cyclic ligand are of great importance, in part because of their research lance to many natural system e.g. , porphyrin and s, calamines publication of several reviews and books covering various aspects of synthetic macro cyclic ligands ${ }^{(1-3)}$

Acyclic model $\mathrm{N}_{2} \mathrm{O}_{2}, \mathrm{~N}_{2} \mathrm{~S}_{2}$ and $\mathrm{N}_{4}$ system derived from 2,6 di acetyl pyridine are used in some chemical processes as catalysts ${ }^{(4,5)}$. They are also used in biological models to understand the structure of bio-molecules ${ }^{(6,7)}$. In the present paper the $\mathrm{Co}(\mathrm{II}), \mathrm{Ni}(\mathrm{II})$ and $\mathrm{Cu}(\mathrm{II})$ complexes with $\mathrm{N} 4$-macrocyclic ligand derived from 2,6-dimethylpyridine and $\mathrm{O}$ phenyline diamine have prepared, fully characterized by spectral and analytical techniques .

\section{Experimental}

\subsection{INSTRUMENTATION:}

IR spectra were recorded using $\mathrm{KBr}$ pellets in the range of $4000-400 \mathrm{~cm}-1$ on a shimadzu spectra photometer ,Micro analyses (C.H.N.) were obtained using a perkin-Elmer 2400 (C.H.N.) Micro analyzer .Electronic spectra absorptions were made on a shimadzo UV-visible spectrophotometer $160 \mathrm{C}$ model for (10-3-10-4 M) solution of $\mathrm{N} 4$ ligand and metal complexes in DMSO and absolute ethanol solvents. The $\mathrm{H} 1 \mathrm{nmr}$ of the N4 ligand chelate was tested on Bruker $300 \mathrm{MHZ}$ $1 \mathrm{H} \mathrm{nmr} \mathrm{spectrophotometer} \mathrm{at} \mathrm{Al-albiat} \mathrm{university} \mathrm{in} \mathrm{Jordan} \mathrm{using} \mathrm{d6-DMSO} \mathrm{solvent} \mathrm{and} \mathrm{TMS} \mathrm{calibrate} \mathrm{.}$

\subsection{MATERIALS:}

\section{Reagents were supplied from Fluka, BDH chemicals.}

\section{A- Preparation of the ligand [L]:}

A $20 \mathrm{ml}$ methanolic solution of 2,6-diacetyl pyridine $(0.01$ mole $)$ was refluxed with $100 \mathrm{ml}$ methanolic solution of re crystallized O-phenyleneiamine $(0.02$ mole) for about $4 \mathrm{hrs}$. A few drops of glacial acetic acid were added to the mixture and refluxed continued for (12-24) hrs. The mixture was concentrated to half of its volume and kept in desiccators for days. The ligand was filtered, washed with methanol, acetone and ether, dried in vacuum, over CaCl2 pellets, in yield $80 \%$, Scheme(1)The physical properties and analytical data, are shown in "Table 1".

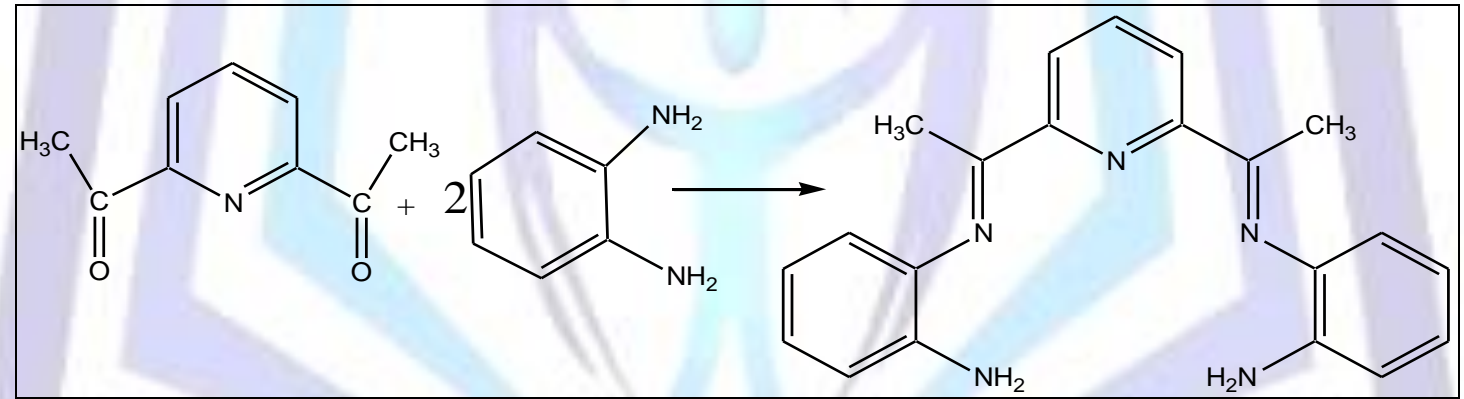

Scheme 1 : synthesis of acyclic ligand $\mathrm{N}_{5}[L]$.

\section{B-General procedure for preparation of complexes :}

The preparation of complexes was carried according to the method published in literature (8), $(0.344 \mathrm{gm}, 1 \mathrm{mmole})$ of N5 ligand in $(10 \mathrm{ml})$ absolute ethanol was added drop wise with stirring to a solution of $1 \mathrm{mmole}$ of $(\mathrm{CoCl} 2.6 \mathrm{H} 2 \mathrm{O}, 0.236 \mathrm{gm})$ in absolute ethanol $(10 \mathrm{ml})$. The reaction were carried out for 24-36 hrs and colored precipitate formed . These were filtered off washed with ethanol, petroleum ether and dried on air ; yield : $70 \%$ [Co( L) Cl2]. A similar method was used to prepare, $(\mathrm{NiCl} 2.6 \mathrm{H} 2 \mathrm{O}, 0.237 \mathrm{gm}),(\mathrm{CuCl} 2.2 \mathrm{H} 2 \mathrm{O}, 0.170 \mathrm{gm})$, yields: $80 \%(\mathrm{Ni} \mathrm{LCl} 2)$ and $81 \%(\mathrm{Cu} \mathrm{L} \mathrm{Cl} 2)$. The physical properties of metal complexes are shown in "Table 1".

\section{Result and Discussion}

\subsection{Stochiometric Study:}

The estimation of the complex structure was carried out by using mole-ratio method in spectrophotometric technique. The results were referred to the (M:L) ratios for all synthesized complexes were(1:1).

\subsection{Conductivity measurement}

The conductivity for $10-3 \mathrm{M}$ solutions of metal complexes Table (3) in (DMSO), show that $\mathrm{Co}$ (II), $\mathrm{Ni}(\mathrm{II})$ and $\mathrm{Cu}$ (II) to be their (1:1) electrolytic (9). 


\subsection{IR and ${ }^{1} \mathrm{H}-\mathrm{NMR}$ Spectra:}

The IR spectra of the N4 ligand and its metal complexes provide information about the metal-ligand bonding. The medium doublet band in the region $3389-3325 \mathrm{~cm}-1$ are assigned to $-\mathrm{NH} 2$ group $(10)$. The strong absorption bands at $1637 \mathrm{~cm}-1$ and $1589-1541 \mathrm{~cm}-1$ are assigned to azomethine groups of $\mathrm{C}=\mathrm{N}$ (terminal) and $\mathrm{C}=\mathrm{N}$ of pyridine ring (8) . These bands are shifted to lower wave number in the spectra of complexes $(1620-1631 \mathrm{~cm}-1)$, which confirms the coordination of nitrogen atoms of $\mathrm{C}=\mathrm{N}$ and nitrogen pyridine ring to metal ion via formation of six-membered ring , that is kinetically stable (3) . The change is intensity and sharp of a $\square \mathrm{NH} 2$ absorptions in the $3300-3400 \mathrm{~cm}-1$ revels participation of $\mathrm{NH} 2$ group in coordination with metal ions understand y therefore, the acyclic ligand behaviors pentadentate of N5- system . Weak absorption bands in the far red regions $400-600 \mathrm{~cm}-1$. The spectra of all metal complexes, provides good indication for M-N band(11).

The proton nmr spectroscopy is a helpful tool for identification of organic compounds in conjugation with other spectrometric information's.

"Figure 1" shows 1HNMR of free ligand in d6-DMSO solvent. 1HNMR spectra of the ligand displayed signals corresponding to the various proton of aromatic of benzyl and pyridine ring, as well as the de shielded absorptions of $\mathrm{NH} 2$ protons $(4 \mathrm{H})$ in (6-6.5) ppm region investigator the condensation of one mole of 2,6-diacetylpyridine with two moles of ophenylenediamine to form acyclic ligand of N5 type(13).

The singlet absorption in the 2-2.5ppm regions investigates as six hydrogen atoms of methyl groups attached to positions 2 and 6 of pyridine ring(14).

A multiple absorptions of chemical shift in the 7-8 ppm regions could be assigned to aromatic Ar- $\mathrm{H}$ and three protons of pyridine rig respectively (15).

The absence of any coupling interactions by $\mathrm{H}-\mathrm{N}$ free groups due to the lack of availability of $\mathrm{H}$ beighbouring atoms render singlet peaks for imino $-\mathrm{C}=\mathrm{N}$ protons(15).

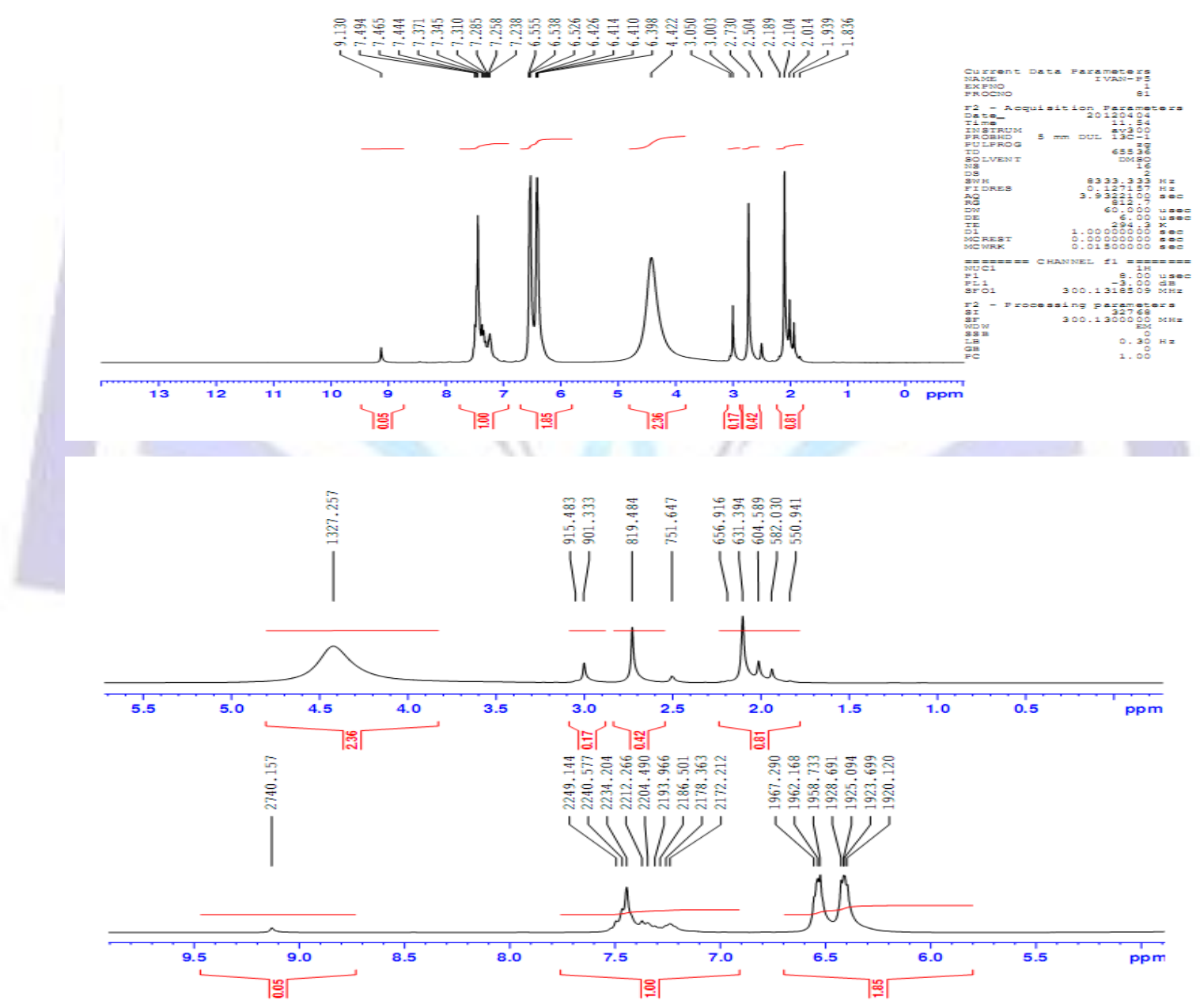

Fig 1: ${ }^{1} \mathrm{H}$-NMR spectrum of ligand in $\mathrm{d}^{6}$-DMSO. 


\subsection{UV-Visible spectra}

The electronic spectra of the ligand and metal complexes were recorded in absorbent ethanol and DMSO solution respectively, The ligand exhibits bands at 234 and $266 \mathrm{~nm}$, there are related to $\square \rightarrow \square^{*}$ and $n \rightarrow \square \square^{*}$ transition of $\mathrm{C}=\mathrm{N}$, $\mathrm{C}=\mathrm{C}$ chromophours in the free ligand. The spectra of the complexes show bands in visible regions of spin-allowed transitions. As well as the $\mathrm{Co}(\mathrm{II}), \mathrm{Ni}(\mathrm{II})$ and $\mathrm{Cu}(\mathrm{II})$ complexes showed weak absorption peaks in the visible region corresponding to the octahedral environments around ions which investigation its octahedral geometry from elemental analysis and mass spectra(16,17).

\subsection{The magnetic moments}

The magnetic moments of divalent Cobalt, nickel, and copper complexes lie in the 3.70, 2.25 and $1.30 \mathrm{BM}$ ranges respectively at room temperature and are close to the predicted value for octahedral geometry around the metal atom (16).

\subsection{The proposed structure}

The data obtained from UN-Visbile and FT- IR spectra, along with expected magnetic moment for all complexes supports the high spin geometry of $\mathrm{Co}(\mathrm{II}), \mathrm{Ni}(\mathrm{II})$ and $\mathrm{Cu}(\mathrm{II})$ complexes $(17,18)$.

It is obvious, observed that free ligand of N5 system differs from N6-system, percent in literature (19-21), where the reaction conditions of metal salts sported coordination with N4 system proceeds rapidly, rather than template system, this facts has investigation the basis of data obtained from 1HNMR, FT-IR., UV-Vis., and others measurements.

A according to the elemental analysis, IR, UV-Visible spectra and magnetic moment of complexes, all the complexes could have the proposed structure in "Figure 2" where the ligand behavior pentadantate of N5 system.

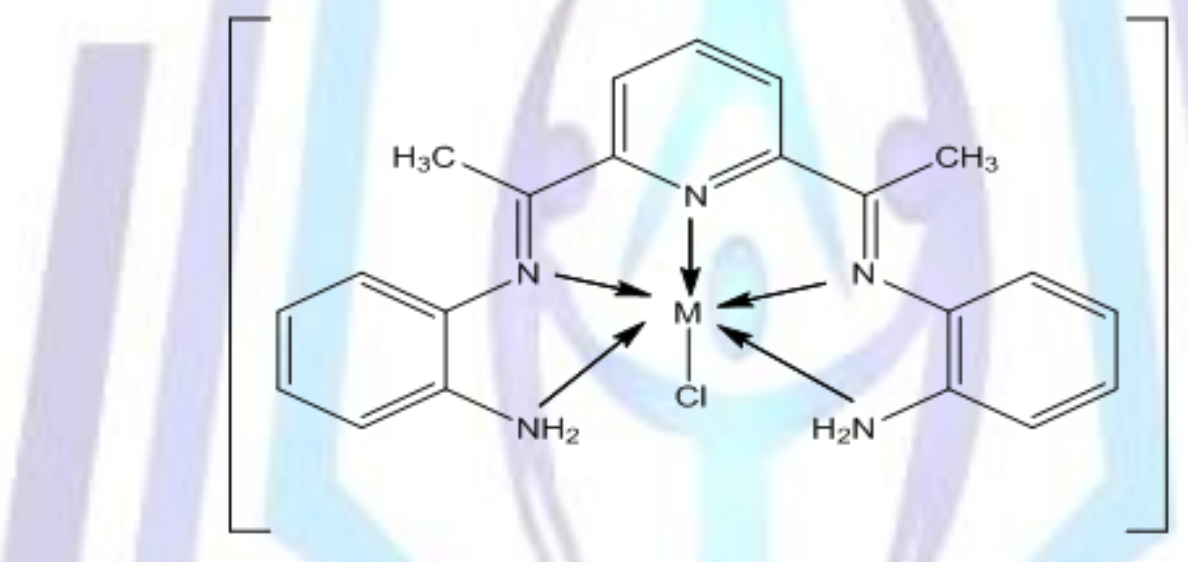

Fig 2 : The proposed structure of Metal complexes

\subsection{Theoretical Study:}

The ball and cylinders and some of selected structural parameters (bond length and angles) of the optimized geometries are shown in "Table 4" ," Figure 3". As shown in this figure, there is no obvious trend for the variation of these parameters. The values of the bond length and angles of the optimized geometries are quite similar to the experimental results of the corresponding compounds. 


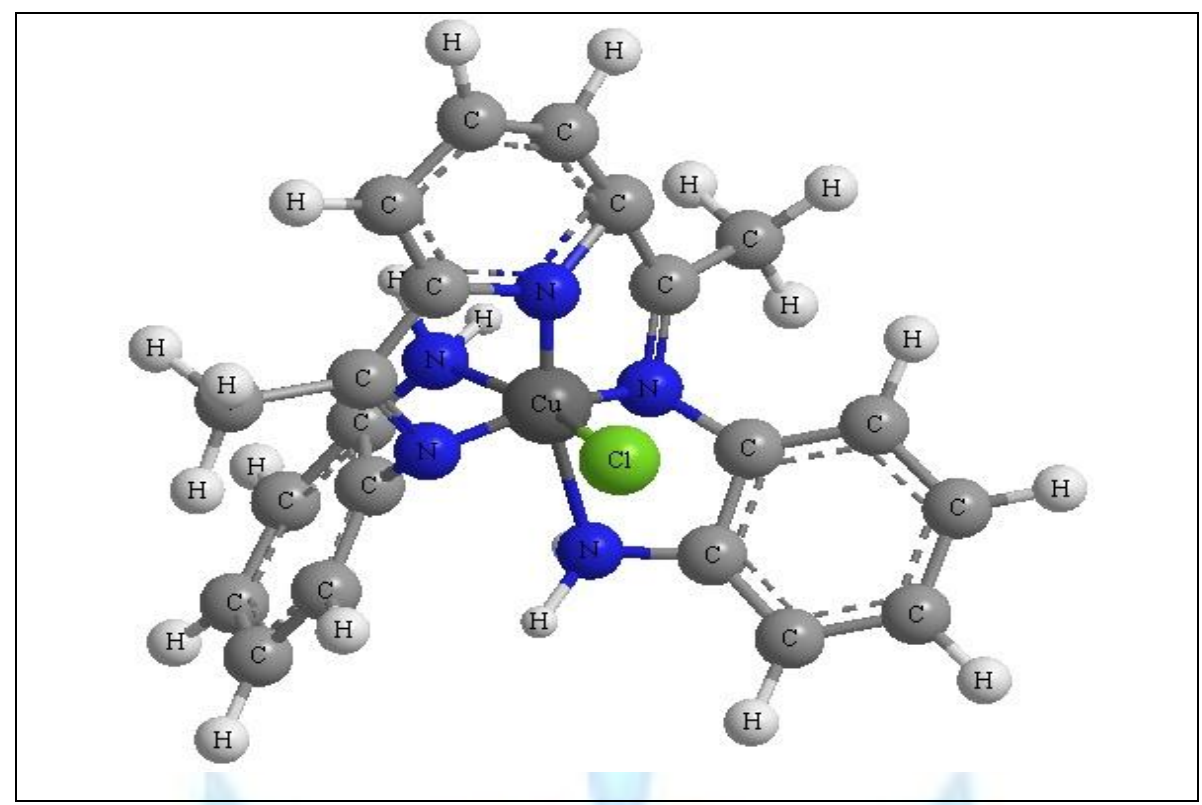

Fig 3 : The optimized structural geometry of $\mathrm{Cu}(\mathrm{II})$ complex

Table 1: Micro Elemental analysis (C.H.N.M) data of ligand

\begin{tabular}{|l|l|l|l|l|l|l|l|}
\hline coumpound & $\begin{array}{l}\text { Molecula } \\
\mathbf{r} \\
\text { formula }\end{array}$ & $\begin{array}{l}\text { M.p. } \\
{ }^{\circ} \mathbf{C}\end{array}$ & color & $\begin{array}{l}\text { \%C } \\
\text { Calc. } \\
\text { (Found) }\end{array}$ & $\begin{array}{l}\text { \%H } \\
\text { Calc. } \\
\text { (Found) }\end{array}$ & $\begin{array}{l}\text { \%N } \\
\text { Calc. } \\
\text { (Found) }\end{array}$ & $\begin{array}{l}\text { \%etal Calc. } \\
\text { (Found) }\end{array}$ \\
\hline $\mathrm{L}$ & 343 & $\begin{array}{l}225- \\
227\end{array}$ & brown & $\begin{array}{l}746.4 \\
(44.31)\end{array}$ & $\begin{array}{l}6.12 \\
(5.81)\end{array}$ & $\begin{array}{l}20.40 \\
(19.71)\end{array}$ & - \\
\hline$[\mathrm{CoLCl}] \mathrm{Cl}$ & 473 & 280 & $\begin{array}{l}\text { Pale } \\
\text { violet }\end{array}$ & $\begin{array}{l}53.29 \\
(51.21)\end{array}$ & $\begin{array}{l}4.43 \\
(3.93)\end{array}$ & $\begin{array}{l}14.66 \\
(14.96)\end{array}$ & $\begin{array}{l}13.30 \\
(12.11)\end{array}$ \\
\hline$[\mathrm{NiLCl}] \mathrm{Cl}$ & 472 & 273 & $\begin{array}{l}\text { Pale } \\
\text { green }\end{array}$ & $\begin{array}{l}41.70 \\
(42.33)\end{array}$ & $\begin{array}{l}3.475 \\
(3.61)\end{array}$ & $\begin{array}{l}(15.09) \\
11.03\end{array}$ \\
\hline$[\mathrm{CuLCl}] \mathrm{Cl}$ & 477 & 295 & blue & $\begin{array}{l}52.78 \\
(50.88)\end{array}$ & $\begin{array}{l}14.66 \\
(14.87)\end{array}$ & 13.30 \\
\hline
\end{tabular}

Table 2: Infrared spectral bands $\left(\mathrm{cm}^{-1}\right)$ and their assignments in the ligand and metal complexes

\begin{tabular}{|l|l|l|l|l|l|}
\hline compound & $v^{(\mathrm{C}=\mathrm{N})}$ & $v^{(\mathrm{NH} 2)}$ & $\begin{array}{l}v^{(\mathrm{C}-\mathrm{N})} \\
v^{(\mathrm{C}=\mathrm{N})(\mathrm{py})}\end{array}$ & $v^{(\mathrm{M}-\mathrm{N})}$ & - \\
\hline $\mathrm{L}$ & $1637(\mathrm{~s})$ & $3389-365(\mathrm{~m})$ & $1589-1600$ & - & \\
\hline$[\mathrm{CoLCl}] \mathrm{Cl}$ & $1631-1591(\mathrm{~s})$ & $3549-3525(\mathrm{~m})$ & $1558-1521(\mathrm{~s})$ & $460-530(\mathrm{~m})$ & $295(\mathrm{~W})$ \\
\hline$[\mathrm{NiLCl}] \mathrm{Cl}$ & $1620(\mathrm{~s})$ & $3564-3408(\mathrm{br})$ & $1575-1562(\mathrm{~s})$ & $434-484(\mathrm{w})$ & $360(\mathrm{w})$ \\
\hline$[\mathrm{CuLCl}] \mathrm{Cl}$ & $1654(\mathrm{~s})$ & $3379(\mathrm{br})$ & $1559(\mathrm{~s})$ & $534-462$ & $310(\mathrm{w})$ \\
\hline
\end{tabular}

$\mathrm{W}=$ weak, br=broad, $\mathrm{s}=$ strong, and $\mathrm{m}=$ medium 
Table 3: Electronic spectra, magnetic moments and molar conductance of the prepared complexes

\begin{tabular}{|c|c|c|c|c|}
\hline Compound & $\begin{array}{l}\lambda_{\max } \\
(\mathrm{nm})\end{array}$ & assignment & $\begin{array}{l}\mu_{\text {eff. }} \\
\text { BM }\end{array}$ & $\begin{array}{l}{ }^{\star \Delta M} \underset{\left.\mathrm{cm}^{2} \mathrm{~mol}^{-1}\right)}{\left(\Omega^{-1}\right.} \\
\end{array}$ \\
\hline L & $\begin{array}{l}266 \\
225\end{array}$ & $\begin{array}{l}\mathrm{n} \rightarrow \pi^{*}, \\
\pi \rightarrow \pi^{*}\end{array}$ & - & - \\
\hline$[\mathrm{CoLCl}] \mathrm{Cl}_{2}$ & $\begin{array}{l}288 \\
404 \\
470\end{array}$ & $\begin{array}{l}{ }^{4} \mathrm{~T}_{1} \mathrm{~g}(\mathrm{~F}) \rightarrow{ }^{4} \mathrm{~T}_{2} \mathrm{~g} \\
{ }^{4} \mathrm{~T}_{1} \mathrm{~g} \rightarrow \mathrm{A}_{2} \mathrm{~g}^{4} \\
{ }^{4} \mathrm{~T}_{1} \mathrm{~g}(\mathrm{~F}) \rightarrow{ }^{4} \mathrm{~T}_{1} \mathrm{~g}(\mathrm{p})\end{array}$ & 3.79 & 73 \\
\hline$[\mathrm{NiLCl}] \mathrm{Cl}$ & $\begin{array}{l}359 \\
377 \\
470\end{array}$ & $\begin{array}{l}\mathrm{A}_{2} \mathrm{~g}^{3} \rightarrow \mathrm{T}_{2} \mathrm{~g}^{3} \\
\mathrm{~A}_{2} \mathrm{~g}^{3} \rightarrow \mathrm{T}_{1} \mathrm{~g}^{3} \\
\mathrm{~A}_{2} \mathrm{~g}^{3} \rightarrow \mathrm{T}_{1} \mathrm{~g}^{3}(\mathrm{P})\end{array}$ & 2.25 & 65 \\
\hline$[\mathrm{CuLCl}] \mathrm{Cl}_{2}$ & $\begin{array}{l}345 \\
550 \\
590\end{array}$ & $\begin{array}{l}{ }^{2} B_{1} g(F) \rightarrow{ }^{2} B_{1} g(F) \\
{ }^{2} B_{2} g^{2}(F) \rightarrow{ }^{2} A_{2} g(p) \\
\text { C.T }\end{array}$ & 1.30 & 82 \\
\hline
\end{tabular}

${ }^{*}=$ molar conductance in $0.001 \mathrm{M}$ solutions in DMSO,C.T=charge transfer of $L$ to $M$ type.

Table 4 : structural parameters, bond length (ㅇ) and angles( $\left.{ }^{\circ}\right)$ of the $[\mathrm{Cu}(\mathrm{L}) \mathrm{Cl}] \mathrm{Cl}$ complex.

\begin{tabular}{|c|c|c|c|c|c|}
\hline \multirow{2}{*}{\multicolumn{2}{|c|}{$\begin{array}{l}\text { Parameters } \\
\text { Bond lengths (ํㅜ) }\end{array}$}} & \multicolumn{2}{|c|}{ Parameters } & \multicolumn{2}{|l|}{ Parameters } \\
\hline & & \multicolumn{2}{|c|}{ Bond angles( $\left(^{\circ}\right)$} & \multicolumn{2}{|l|}{ Bond angles $(\stackrel{\circ}{)})$} \\
\hline $\mathrm{Cu}(27)-\mathrm{Cl}(28)$ & 2.1600 & $\mathrm{Cu}(27)-\mathrm{Cl}(28)$ & 2.1600 & $C(18)-C(17)-C(16)$ & 119.9986 \\
\hline$N(26)-H(47)$ & 1.0500 & $\mathrm{~N}(26)-\mathrm{H}(47)$ & 1.0500 & $H(39)-C(16)-C(17)$ & 120.0014 \\
\hline$N(26)-C u(27)$ & 1.8460 & $\mathrm{~N}(26)-\mathrm{Cu}(27)$ & 1.8460 & $H(39)-C(16)-C(15)$ & 120.0007 \\
\hline$C(25)-H(46)$ & 1.1000 & $\mathrm{C}(25)-\mathrm{H}(46)$ & 1.1000 & $C(17)-C(16)-C(15)$ & 119.9979 \\
\hline$C(24)-C(25)$ & 1.3370 & $C(24)-C(25)$ & 1.3370 & $H(41)-C(18)-C(17)$ & 120.0000 \\
\hline$C(23)-N(26)$ & 1.6241 & $C(23)-N(26)$ & 1.6241 & $H(41)-C(18)-C(13)$ & 119.9999 \\
\hline$C(23)-C(24)$ & 1.3370 & $C(23)-C(24)$ & 1.3370 & $C(17)-C(18)-C(13)$ & 120.0001 \\
\hline$C(22)-H(45)$ & 1.1000 & $\mathrm{C}(22)-\mathrm{H}(45)$ & 1.1000 & $H(38)-C(15)-C(16)$ & 119.9999 \\
\hline$C(22)-C(23)$ & 1.3370 & $C(22)-C(23)$ & 1.3370 & $H(38)-C(15)-C(14)$ & 120.0003 \\
\hline$C(21)-H(44)$ & 1.1000 & $\mathrm{C}(21)-\mathrm{H}(44)$ & 1.1000 & $C(16)-C(15)-C(14)$ & 119.9998 \\
\hline$C(21)-C(22)$ & 1.3370 & $C(21)-C(22)$ & 1.3370 & $N(19)-C(14)-C(15)$ & 107.0733 \\
\hline$C(20)-H(43)$ & 1.1000 & $\mathrm{C}(20)-\mathrm{H}(43)$ & 1.1000 & $N(19)-C(14)-C(13)$ & 118.4057 \\
\hline$C(25)-C(20)$ & 1.3370 & $C(25)-C(20)$ & 1.3370 & $C(15)-C(14)-C(13)$ & 120.0001 \\
\hline$C(20)-C(21)$ & 1.3377 & $C(20)-C(21)$ & 1.3377 & $\mathrm{H}(47)-\mathrm{N}(26)-\mathrm{Cu}(27)$ & 110.8667 \\
\hline$N(19)-H(42)$ & 1.0500 & $\mathrm{~N}(19)-\mathrm{H}(42)$ & 1.0500 & $H(47)-N(26)-C(23)$ & 110.8675 \\
\hline $\mathrm{N}(19)-\mathrm{Cu}(27)$ & 1.8460 & $N(19)-C u(27)$ & 1.8460 & $\mathrm{Cu}(27)-\mathrm{N}(26)-\mathrm{C}(23)$ & 138.2659 \\
\hline$C(18)-H(41)$ & 1.1000 & $\mathrm{C}(18)-\mathrm{H}(41)$ & 1.1000 & $\mathrm{H}(42)-\mathrm{N}(19)-\mathrm{Cu}(27)$ & 135.7281 \\
\hline$C(17)-H(40)$ & 1.1000 & $\mathrm{C}(17)-\mathrm{H}(40)$ & 1.1000 & $H(42)-N(19)-C(14)$ & 135.7282 \\
\hline$C(17)-C(18)$ & 1.3370 & $C(17)-C(18)$ & 1.3370 & $\mathrm{Cu}(27)-\mathrm{N}(19)-\mathrm{C}(14)$ & 88.5436 \\
\hline$C(16)-H(39)$ & 1.1000 & $C(16)-H(39)$ & 1.1000 & $C(25)-C(24)-C(23)$ & 119.9979 \\
\hline$C(16)-C(17)$ & 1.3371 & $C(16)-C(17)$ & 1.3371 & $C(25)-C(24)-N(12)$ & 128.9982 \\
\hline$C(15)-H(38)$ & 1.1000 & $\mathrm{C}(15)-\mathrm{H}(38)$ & 1.1000 & $C(23)-C(24)-N(12)$ & 111.0004 \\
\hline$C(15)-C(16)$ & 1.3370 & $C(15)-C(16)$ & 1.3370 & $H(37)-C(11)-H(36)$ & 109.5200 \\
\hline$C(14)-N(19)$ & 1.1762 & $C(14)-N(19)$ & 1.1762 & $H(37)-C(11)-H(35)$ & 109.4618 \\
\hline
\end{tabular}




\begin{tabular}{|c|c|c|c|c|c|c|}
\hline$C(14)-C(15)$ & 1.3370 & $C(14)-C(15)$ & 1.3370 & $\mathrm{H}(37)-\mathrm{C}(11)-\mathrm{C}(9)$ & 109.4619 & \\
\hline$C(18)-C(13)$ & 1.3370 & $C(18)-C(13)$ & 1.3370 & $H(36)-C(11)-H(35)$ & 109.4416 & \\
\hline$C(13)-C(14)$ & 1.3370 & $C(13)-C(14)$ & 1.3370 & $H(36)-C(11)-C(9)$ & 109.4422 & \\
\hline $\mathrm{N}(12)-\mathrm{Cu}(27)$ & 1.3030 & $\mathrm{~N}(12)-\mathrm{Cu}(27)$ & 1.3030 & $H(35)-C(11)-C(9)$ & 109.4999 & \\
\hline$C(24)-N(12)$ & 1.2600 & $C(24)-N(12)$ & 1.2600 & $\mathrm{Cu}(27)-\mathrm{N}(12)-\mathrm{C}(24)$ & 104.0000 & \\
\hline$C(11)-H(37)$ & 1.1130 & $\mathrm{C}(11)-\mathrm{H}(37)$ & 1.1130 & $\mathrm{Cu}(27)-\mathrm{N}(12)-\mathrm{C}(9)$ & 93.4067 & \\
\hline$C(11)-H(36)$ & 1.1130 & $C(11)-H(36)$ & 1.1130 & $C(24)-N(12)-C(9)$ & 114.9999 & \\
\hline$C(11)-H(35)$ & 1.1130 & $C(11)-H(35)$ & 1.1130 & $C(18)-C(13)-C(14)$ & 119.9984 & \\
\hline$C(10)-H(34)$ & 1.1130 & $C(10)-H(34)$ & 1.1130 & $C(18)-C(13)-N(8)$ & 128.9982 & \\
\hline$C(10)-H(33)$ & 1.1130 & $C(10)-H(33)$ & 1.1130 & $C(14)-C(13)-N(8)$ & 110.9999 & \\
\hline$C(10)-H(32)$ & 1.1130 & $\mathrm{C}(10)-\mathrm{H}(32)$ & 1.1130 & $H(34)-C(10)-H(33)$ & 109.5204 & \\
\hline$C(9)-N(12)$ & 2.0384 & $C(9)-N(12)$ & 2.0384 & $H(34)-C(10)-H(32)$ & 109.4616 & \\
\hline$C(9)-C(11)$ & 1.4970 & $C(9)-C(11)$ & 1.4970 & $H(34)-C(10)-C(7)$ & 109.4620 & \\
\hline $\mathrm{N}(8)-\mathrm{Cu}(27)$ & 1.3030 & $\mathrm{~N}(8)-\mathrm{Cu}(27)$ & 1.3030 & $H(33)-C(10)-H(32)$ & 109.4417 & \\
\hline $\mathrm{C}(13)-\mathrm{N}(8)$ & 1.2600 & $C(13)-N(8)$ & 1.2600 & $H(33)-C(10)-C(7)$ & 109.4418 & \\
\hline$C(7)-C(10)$ & 1.4970 & $C(7)-C(10)$ & 1.4970 & $H(32)-C(10)-C(7)$ & 109.4999 & \\
\hline$C(7)-N(8)$ & 1.9464 & $C(7)-N(8)$ & 1.9464 & $\mathrm{Cu}(27)-\mathrm{N}(8)-\mathrm{C}(13)$ & 104.0002 & \\
\hline$C(6)-H(31)$ & 1.1000 & $\mathrm{C}(6)-\mathrm{H}(31)$ & 1.1000 & $\mathrm{Cu}(27)-\mathrm{N}(8)-\mathrm{C}(7)$ & 97.7309 & \\
\hline$C(5)-H(30)$ & 1.1000 & $C(5)-H(30)$ & 1.1000 & $C(13)-N(8)-C(7)$ & 148.5777 & \\
\hline$C(5)-C(6)$ & 1.0286 & $C(5)-C(6)$ & 1.0286 & $\mathrm{H}(31)-\mathrm{C}(6)-\mathrm{C}(5)$ & 122.1912 & \\
\hline$C(4)-C(7)$ & 1.3370 & $C(4)-C(7)$ & 1.3370 & $H(31)-C(6)-C(1)$ & 122.1914 & \\
\hline$C(4)-C(5)$ & 1.3370 & $C(4)-C(5)$ & 1.3370 & $C(5)-C(6)-C(1)$ & 115.6173 & \\
\hline $\mathrm{N}(3)-\mathrm{Cu}(27)$ & 1.3030 & $\mathrm{~N}(3)-\mathrm{Cu}(27)$ & 1.3030 & $C(10)-C(7)-N(8)$ & 137.2290 & \\
\hline$N(3)-C(4)$ & 1.2600 & $N(3)-C(4)$ & 1.2600 & $C(10)-C(7)-C(4)$ & 137.2291 & \\
\hline$C(2)-C(9)$ & 1.3370 & $C(2)-C(9)$ & 1.3370 & $N(8)-C(7)-C(4)$ & 85.5420 & \\
\hline$C(2)-N(3)$ & 1.2600 & $C(2)-N(3)$ & 1.2600 & $H(30)-C(5)-C(6)$ & 115.3087 & \\
\hline$C(1)-H(29)$ & 1.1000 & $C(1)-H(29)$ & 1.1000 & $H(30)-C(5)-C(4)$ & 115.3086 & \\
\hline$C(6)-C(1)$ & 1.3370 & $C(6)-C(1)$ & 1.3370 & $C(6)-C(5)-C(4)$ & 129.3826 & \\
\hline \multirow[t]{16}{*}{$C(1)-C(2)$} & 1.3370 & $C(1)-C(2)$ & 1.3370 & $\mathrm{Cl}(28)-\mathrm{Cu}(27)-\mathrm{N}(26)$ & & 53.2011 \\
\hline & & $H(44)-C(21)-C(22)$ & 120.0177 & $\mathrm{Cl}(28)-\mathrm{Cu}(27)-\mathrm{N}(19)$ & & 47.3262 \\
\hline & & $H(44)-C(21)-C(20)$ & 120.0172 & $\mathrm{Cl}(28)-\mathrm{Cu}(27)-\mathrm{N}(12)$ & & 50.5084 \\
\hline & & $C(22)-C(21)-C(20)$ & 119.9652 & $\mathrm{Cl}(28)-\mathrm{Cu}(27)-\mathrm{N}(8)$ & 63.0505 & \\
\hline & & $H(43)-C(20)-C(25)$ & 120.0131 & $\mathrm{Cl}(28)-\mathrm{Cu}(27)-\mathrm{N}(3)$ & 96.4633 & \\
\hline & & $H(43)-C(20)-C(21)$ & 120.0132 & $\mathrm{~N}(26)-\mathrm{Cu}(27)-\mathrm{N}(19)$ & 5.9331 & \\
\hline & & $C(25)-C(20)-C(21)$ & 119.9737 & $\mathrm{~N}(26)-\mathrm{Cu}(27)-\mathrm{N}(12)$ & 5.9330 & \\
\hline & & $H(46)-C(25)-C(24)$ & 119.9996 & $N(26)-C u(27)-N(8)$ & 109.5001 & \\
\hline & & $H(46)-C(25)-C(20)$ & 120.0002 & $\mathrm{~N}(26)-\mathrm{Cu}(27)-\mathrm{N}(3)$ & 109.5000 & \\
\hline & & $C(24)-C(25)-C(20)$ & 120.0003 & $\mathrm{~N}(19)-\mathrm{Cu}(27)-\mathrm{N}(12)$ & 6.6846 & \\
\hline & & $H(45)-C(22)-C(23)$ & 120.0002 & $\mathrm{~N}(19)-\mathrm{Cu}(27)-\mathrm{N}(8)$ & 104.0000 & \\
\hline & & $\mathrm{H}(45)-\mathrm{C}(22)-\mathrm{C}(21)$ & 120.0000 & $\mathrm{~N}(19)-\mathrm{Cu}(27)-\mathrm{N}(3)$ & 109.5000 & \\
\hline & & $C(23)-C(22)-C(21)$ & 119.9997 & $\mathrm{~N}(12)-\mathrm{Cu}(27)-\mathrm{N}(8)$ & 109.4998 & \\
\hline & & $\mathrm{N}(26)-\mathrm{C}(23)-\mathrm{C}(24)$ & 40.4207 & $\mathrm{~N}(12)-\mathrm{Cu}(27)-\mathrm{N}(3)$ & 104.0000 & \\
\hline & & $N(26)-C(23)-C(22)$ & 160.4180 & $\mathrm{~N}(8)-\mathrm{Cu}(27)-\mathrm{N}(3)$ & 104.0001 & \\
\hline & & $C(24)-C(23)-C(22)$ & 119.9999 & $C(7)-C(4)-C(5)$ & 128.9987 & \\
\hline
\end{tabular}




\section{REFERENCES}

[1] Tyagi M. and Chandra. 2012. ORen Journal of Inorg.Chem.2, pp 41-48

[2] Mishra, D. Nastar S., Drew, M.G.B. and Gattopadhyay S.K. 2006. Inorg. chimica Actr.359, 585-592

[3] Raja N. and Ramesh R. (2010), spectrochimica Acta A, 75, pp.713-718

[4] Chandra S., Tyagi M. and Agrawal , S. 2010. journal of Saudi chem. Soc.15,pp.49- 54.

[5] Sharma K., Singh, Fahnui, N. and Singh ,R.V. 2010. Spectrachimica Acta A. 75,pp. 422-427

[6] FerraZ ,K.O, Waradell, S.M.SV and Beraldo H. .2009. spectrachimica Acta. A,737, Pp.140-145.

[7] D.P. singh, S. Nupar, B.p. Yadar and V. B.rara . 1997. polyhedron.16 (13),pp.2229-2232.

[8] Ali S.A ., soliman, M.M. Aboaly, R.M. Ramadam, J.Coord.chem.55. 2002.1161

[9] Solooman , L..D, Kessling , G. Mobilitition of cation -macrocyclic Ligand complexes , pure Appl. Chem.1993. 65(7), 1533.

[10] Sliverstin R.M. ,G.c.Bassler, T.C. Morill, spectroscopic Identification of organic compounds 4thed.,Wiley, New York. 1981.

[11] Nakmato K., Infrared and Raman spectra of Inorganic and coordination compounds, $4^{\text {th }}$ ed., Wiley, New York. 1986.

[12] Ferraro, J.R "Low frequency Vibration of Inorganic and coordination compound Plenum press, New York.1977.

[13] Ayed, S.al. Shilri. 2004. Synthesis, characterization and thermal analysis of some new metal complexes of poly dentate Schiff base, spectra chimica Acta, part A, 60, 1189-1192.

[14] Williams D.H. ,I.Fleming,Spectroscopic Methods in Organic Compounds, $4^{\text {th }}$. Ed. Mc.Graw-Hill.1989. London.

[15] Sreekanth,M.R.P.Kurup,Spectrochim.Acta,59A. 2003.1349.

[16] Lever A.B.P. "Electronic spectra of Inorganic and coordination compounds "Elsevier. 1968.

[17] wived D., r., Sing V.Falimi N, Singh R.V. 2003. Int. Chem. J. Sci, (2)p.233.

[18] Josey R.S. phus.C.J. Dhanara j and M.S Nair Transition Met Chem. 2006. 31, 699.

[19] Canpolat E.A. Y. and M.Kayes, transition .Met.Chem. 2006. 31, 653.

[20] Clark, R.J.H. and Williams., C.S. Inorganic chem. 1965. 4,350.

[21] Singh D. P. and et al . polyhedron. 1977. 16(13) pp.2229-2232. 